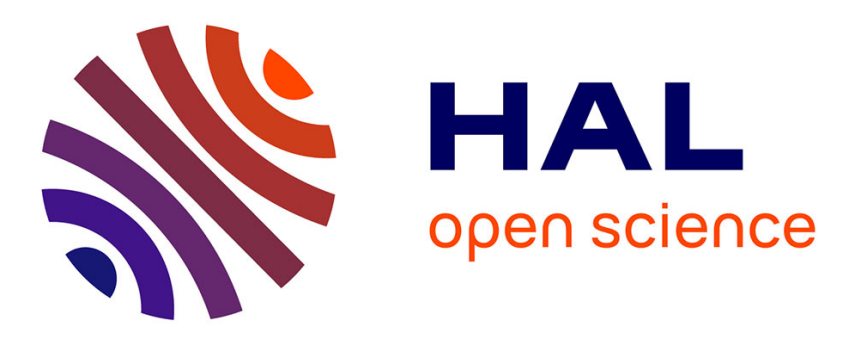

\title{
Lignes de failles générationnelles à propos des enfants juifs cachés en France pendant la seconde guerre mondiale
}

\author{
Marion Feldman
}

\section{- To cite this version:}

Marion Feldman. Lignes de failles générationnelles à propos des enfants juifs cachés en France pendant la seconde guerre mondiale. Cahiers de psychologie clinique, 2020, Violence de masse, $\mathrm{n}^{\circ} 54$ (1), pp.115-130. 10.3917/cpc.054.0115 . hal-03131816

\section{HAL Id: hal-03131816 \\ https://hal.parisnanterre.fr/hal-03131816}

Submitted on 4 Feb 2021

HAL is a multi-disciplinary open access archive for the deposit and dissemination of scientific research documents, whether they are published or not. The documents may come from teaching and research institutions in France or abroad, or from public or private research centers.
L'archive ouverte pluridisciplinaire HAL, est destinée au dépôt et à la diffusion de documents scientifiques de niveau recherche, publiés ou non, émanant des établissements d'enseignement et de recherche français ou étrangers, des laboratoires publics ou privés. 


\title{
LIGNES DE FAILLES GÉNÉRATIONNELLES À PROPOS DES ENFANTS JUIFS CACHÉS EN FRANCE PENDANT LA SECONDE GUERRE MONDIALE
}

\author{
Marion FELDMAN ${ }^{1}$
}

RéSUMÉ Cet article se propose d'identifier les modalités de transmission des traumatismes à leurs enfants vécus par les enfants juifs cachés pendant la Seconde Guerre mondiale. Après avoir rappelé le vécu si singulier de ces enfants devenus adultes, puis à travers l'analyse d'une situation de recherche clinique, nous montrons les processus à l'œuvre. Le premier concerne un vécu douloureux avec une transmission ambivalente du mortifère et des pulsions de vie ; le second correspond au fait que les enfants juifs cachés ont représenté une génération particulière ayant un vécu spécifique. La discussion s'articule autour des notions importantes de filiation, de transmission d'une crypte et d'un héritage transgénérationnel complexe mais également de la contrainte à la transformation et à la narrativité.

MoTS-CLÉS enfants juifs cachés, transmission, transgénérationnel, trauma, héritage, narrativité.

1 Professeure de Psychopathologie Psychanalytique - Université Paris Nanterre, EA4430 CLIPSYD - A2P, Approches en Psychopathologie psychanalytique, psychologue-clinicienne. m.feldman@ parisnanterre.fr 


\section{GENERATIONAL FAULT LINES STEMMING FROM JEWISH CHILDREN WHO WERE HIDDEN DURING THE SECOND WORLD WAR}

ABSTRACT This article attempts to understand how the experience of being a Jewish child hidden in France during the Occupation is transmitted between generations. After reflecting on the unique experience of these Jewish children, we analyze a clinical research case in order to reveal the specific processes at work in transmission. First, we identify a painful experience that is accompanied by a contradictory transmission of life drives and thoughts about death. Second, we consider the fact that hidden Jewish children represent a very specific generation with a unique experience. The discussion is structured around several different issues including filiation, the transmission of a crypt and a complex transgenerational heritage, and the constraints on transformation and narration.

KEYWORDS hidden Jewish children, transmission, transgenerational, trauma, heritage, narrativity.

\section{Introduction}

Dans son roman Lignes de failles dont l'intitulé prend appui sur la métaphore d'une cassure de la roche, Nancy Huston montre comment un traumatisme vécu à une génération peut venir ébranler les trois suivantes.

En géologie, les lignes de faille désignent des fissures dans une structure de la croûte terrestre où il $\mathrm{y}$ a eu des mouvements. Les tremblements de terre ou les séismes se produisent généralement le long de failles parce qu'elles constituent des zones de fragilité. Ces lignes de faille désignent ainsi les fractures constituées à la petite enfance qui poursuivent les individus de génération en génération. Au travers du regard d'un enfant, et sur quatre générations, l'auteure met également en lumière la représentation qu'a un enfant du monde à l'âge de six ans, et ceci à quatre époques différentes.

A ce propos, Abraham et Torok (1978) ont développé une réflexion particulièrement pertinente sur le transgénérationnel. Ils mettent en avant les modalités de transmission d'un 
trauma d'un parent à son enfant. La symbiose psychique entre la mère et son bébé constitue un premier cadre du "passage" et participe de la construction des premières représentations de l'enfant. Le passage est influencé par le langage verbal dans la famille mais également par la façon dont le bébé va être porté, bercé, nourri (Tisseron, 1995). Ce "berceau" va constituer des sortes d'indices et façonner les représentations de l'enfant, mais aussi ses affects et ses comportements. Chez le parent traumatisé, c'est par le clivage que la "part morte" va se constituer et ne plus pouvoir se défaire dans une sorte de "suspens du traumatisme", soit une impossibilité d'élaboration de cette "part morte silencieusement omniprésente". Il y a alors un empêchement pour l'enfant à opérer une identification projective auprès de son parent. La part du négatif engendré par le parent est alors, possiblement, un support de sa construction psychique. Il transmet en partie son aliénation, son sentiment d'insécurité qui peut prendre le pas sur sa personnalité "culturelle", c'est-à-dire le cadre culturel, externe, dans laquelle il s'est construit. L'enfant risque de devenir alors le contenant de l'histoire parentale à défaut d'en être l'héritier.

Abraham et Torok (1978) ont montré ainsi l'impact des silences et des deuils non accomplis: "Tous les mots qui n'auront pas été dits, toutes les scènes qui n'auront pu être remémorées, toutes les larmes qui n'auront pu être versées, seront avalés, en même temps que le traumatisme cause de la perte. Avalés et mis en conserve. Le deuil indicible installe à l'intérieur du sujet un caveau secret" (p.266). Transmettre à son insu, par le non-dit est un facteur de risque psychopathologique, car le symbolique n'advenant pas, les maux peuvent alors prendre le dessus.

Si un certain nombre d'écrits évoque les processus de transmission à leur descendance des traumatismes chez les survivants de la Shoah, peu d'entre eux précisent la particularité de la transmission du vécu spécifique chez les enfants juifs cachés pendant la Seconde Guerre mondiale.

C'est en prenant appui sur l'histoire d'un des sujets rencontrés dans le cadre d'une recherche clinique (Feldman, 2009), que nous tenterons de décrypter les modalités à l'œuvre dans ce qui pourrait être caractérisé de séisme historique avec des magnitudes plus ou moins importantes selon le contexte et l'histoire singulière de chacun. 


\section{Le vécu des enfants juifs cachés en France pendant la Seconde Guerre mondiale}

Pour échapper à la déportation qui menaçait tous les Juifs d'Europe pendant la Seconde Guerre mondiale, un certain nombre d'enfants ont été contraints de vivre cachés pour assurer leur survie. Ils pouvaient devenir invisibles ou rester visibles (Dwork, 1991), c'est-à-dire qu'ils étaient soit cachés au sens propre du terme, dans un endroit clôt pour demeurer invisible physiquement, soit cachés avec un changement d'identité, de prénom, de nom, de religion, d'environnement, et pour la plupart d'entre eux, séparés de leurs parents. Ainsi, ces enfants ne pouvaient plus être enfants de leurs parents ; ils ne pouvaient plus être juifs, ils ont subi des pertes, des privations, parfois des humiliations et des maltraitances, et des frayeurs multiples (Feldman, 2009). Outre ces traumas, ils ont dû supporter le silence et l'absence de reconnaissance de leur vécu. Enfants, ils se sont construits de façon spécifique, en lien avec cette situation singulière d'avoir été cachés.

Il a fallu attendre l'année 1991, pour que la souffrance des "enfants cachés" soit reconnue officiellement, avec une réunion internationale qui a eu lieu à New-York, présidée par Elie Wiesel, et 1995 pour que le Président de la République française, Jacques Chirac, reconnaisse la responsabilité de la France dans les crimes commis envers les Juifs. Les enfants cachés avaient plus de cinquante ans...

Ces enfants ont grandi avec le silence de la période de clandestinité et de celui de l'après-guerre.

$\mathrm{Au}$ lendemain de la guerre, dans le contexte du « syndrome de Vichy, ou du passé qui ne passe pas » (Conan, Rousseau, 1994), l'important était d'aller de l'avant et surtout ne pas se retourner sur ce qui s'était passé. Les déportés devaient reprendre une vie "normale" au même titre que les autres, et les enfants attendaient soit que leurs parents viennent les chercher, soit que d'autres décisions soient prises, et qu'ils soient dirigés vers des maisons d'enfants ou des familles adoptives. Les quelques parents de retour de déportation, puisqu'ils avaient eu cette chance de revenir des camps, auraient dû retrouver leur progéniture et réjouir l'opinion publique. Or cela n'a pas été le cas. "Les enfants ont été dépositaires d'une histoire que tout le monde voulait oublier, d'une culpabilité 
collective que la majorité des Français voulait garder enfouie" (Poujol, 2006, p.127).

Ces traumatismes liés à ce vécu, ont eux-mêmes souvent été cachés, non divulgués, non partagés avec la famille : conjoint, enfants, petits-enfants, notamment par le fait que la reconnaissance de leur vécu douloureux a été tardive (Feldman, 2016). Ces traumatismes «cumulatifs» définis comme «l'accumulation de défaillances répétées de l'objet maternel dans son rôle de barrière protectrice et de moi auxiliaire » (Khan, 1976, p.74) qui ont fait effraction dans le passé infantile, se sont manifesté, à certains moments précis de la vie psychique (Feldman, 2019) et ont eu des effets sur la descendance.

\section{"La guerre est finie, mais pas la mienne. Pas la mienne..."}

Paulette $^{2}$ est née en 1936, et elle est fille unique. Ses parents sont nés en France ; ses grands-parents viennent de Pologne. À sa naissance, Paulette est placée en pouponnière puis chez une nourrice. Un jour, son grand-père maternel lui rend visite et découvre qu'elle est maltraitée par cette nourrice. Sa mère vient la chercher et décide de s'occuper d'elle. Son père étant soldat, Paulette est, en fait, élevée par sa mère et sa grandmère maternelle. Son père, elle ne le connaîtra qu'en 1945, puisque lorsque la guerre est déclarée, il est fait prisonnier. Sa grand-mère maternelle parle mal le français. Elle parle yiddish à sa petite-fille. Au moment de la guerre, sa mère travaille comme sténo-dactylo, sa grand-mère s'occupe d'enfants. Sa mère travaille pour l'UGIF ${ }^{3}$ : "Comme ça, lui avait-on dit, elle pourrait peut-être protéger elle-même sa famille, c'est-à-dire sa mère et moi".

Sa première grande humiliation, Paulette la vit quand elle est à l'école, en cours préparatoire. Une remise de prix est organisée en fin d'année scolaire. Le "Prix d'excellence" lui est destiné, puisqu'elle est première en classe. Sont alors nommés les prix d'honneur, les autres prix, mais pas elle pour le prix d'excellence : “J'ai été oubliée ? On m'a oubliée ? J'ai fait quelque chose de mal? J'ai rien compris mais là, quelque chose s'est noué en moi". La cérémonie terminée, "ma mère et moi, nous rentrons dans le silence jusqu'à la maison". Au
2 Pour des raisons éthiques, le prénom a été modifié. Paulette, ainsi que les autres sujets rencontrés, m'ont accordé leur consentement à cette recherche.

3 Union générale des Israélites de France. 
retour, sa mère lui offre un cadeau, un livre, en lui disant que c'est à elle que revient le "Prix d'excellence". Paulette raconte ne pas avoir compris ce geste : "J'étais dans une incohérence totale", ce livre, offert à ce moment-là, hors de la cérémonie, ne sanctionne plus aucune excellence. Paulette connaît ensuite une nouvelle incohérence lorsqu'elle doit porter l'Etoile jaune et qu'elle ne le veut pas, sa mère lui dit: " $\mathrm{Si}$, si ça fera joli".

Par la suite, sa mère disparait, rien ne lui est dit. Paulette remarque que sa grand-mère est très angoissée. Sa mère est arrêtée par la Gestapo à Paris. Á ce moment-là, Paulette est cachée avec sa grand-mère maternelle dans une "chambre de bonne", grâce à la concierge de l'immeuble. Un jour, un homme que Paulette ne connait pas, vient la chercher et l'emmène dans la Sarthe : "Moi, je ne dis pas au-revoir à ma grand-mère"; la menace est telle que sa grand-mère ne peut pas effectuer le trajet ou même lui expliquer où elle va et lui dire au-revoir. Paulette raconte qu'à partir de ce moment-là, elle commence à être mutique. Elle se souvient être allée chez une femme qui habitait dans une ferme : "J'étais livrée complètement à moi-même". Paulette dit être restée cloîtrée dans le silence. "J'ai vivoté deux mois, trois mois, peut-être quatre, jusqu'au jour où un matin, je me réveille, la ferme est vide". C'était un nouvel abandon. Elle reste plusieurs jours à errer, toute seule, ne sait pas combien de temps. "J'étais disparue, j'avais disparu", dit-elle.

Elle se retrouve, sans savoir comment dans une autre ferme. Il y a un couple et deux enfants. L'homme est alcoolique : "Il était ivre, violent et il hurlait. Il battait les deux filles". Paulette dit s'être occupée de débarrasser la table, elle ne s'asseyait pas pour manger. Elle se souvient d'avoir mangé les miettes, mais ne se rappelle pas avoir éprouvé la faim : "Je picorais". Elle se rappelle avoir eu la galle et des poux. Elle dort dans la grange, dans le foin. Quand je demande à Paulette les prénoms des deux filles, elle me dit ne pas les connaître, elle ne semble jamais les avoir connues, "puisqu'on ne se parlait pas". Elle-même porte un autre nom. Elle dit d'elle : "J'étais un petit moustique", pour signifier que son existence importe si peu pour cette famille. "J'étais rien" pour personne.

À la Libération, des Américains viennent chercher Paulette. Ils la conduisent dans un hôpital car elle est dénutrie : "Les 
sœurs étaient brutales". Elle reste dans cet hôpital pendant une dizaine de jours. Un homme vient la chercher et la conduit dans château où sont rassemblés d'autres enfants ${ }^{4}$. C'est la première impression positive qu'elle a à ce moment-là, elle dit que cet homme lui parle en la regardant. Elle va à l'école : "Ça a été le début de ma renaissance". Un jour, la grandmère de Paulette vient la chercher au château. Elle retourne à Paris avec elle. Paulette décrit cette période-là de la façon suivante: "La guerre est finie, mais pas la mienne. Pas la mienne".

Elle retourne à l'école, est aidée par une institutrice qui lui fait rattraper son retard. Sa mère rentre des camps : "Elle est revenue malade, physiquement et psychologiquement". Le retour de son père, c'est le retour d'un inconnu, c'est, ditelle, "une catastrophe" : "J'entrais pas du tout dans son sillage de vie... Il ne m'a pas rejetée physiquement, et rejetée moralement". À l'école puis au collège, Paulette dit souffrir d'antisémitisme, mais à la maison, ses parents ne veulent rien savoir de la souffrance de leur fille. Paulette décrit son père comme ayant eu des comportements anormaux vis-à-vis d'elle, notamment un comportement humiliant à son égard, en disqualifiant tout ce qu'elle faisait, tout ce qu'elle était. Sa mère n'a pas les capacités psychiques pour la protéger. Ses parents reprennent leur travail de vendeurs sur les marchés. Paulette mène sa vie entre l'école et les vacances d'été, pendant lesquelles ses parents l'envoient à la campagne dans des familles catholiques, où Paulette dit s'être ennuyée et fréquenter l'église.

Paulette est brillante au lycée, mais à cause d'un "zéro pointé", mis de façon arbitraire par un professeur d'histoire, elle échoue à son baccalauréat. À ce moment-là, Paulette arrête de manger: "Je deviens anorexique, mais personne ne s'en est aperçu". Par l'intermédiaire d'une amie à elle, elle intègre un mouvement juif de jeunesse. Elle passe un diplôme professionnel de secrétaire de direction.

Paulette tente de partir en Israël, mais se dit peureuse de tout. Elle rencontre un homme, un Israélien, à Paris. C'est le premier homme à lui prêter attention. Quand Paulette tombe enceinte, son père l'oblige à se marier. L'union est célébrée à la synagogue. Elle a un deuxième enfant avec lui. Alors que son fils a cinq ans, et sa fille quatre ans, Paulette divorce,
4 Château qui appartenait à l'OSE "C'est un aumônier juif, de l'armée américaine qui se démène pour loger au château de Méhoncourt, dans la Sarthe, une trentaine d'enfants abandonnés. L'OSE prend le relais et y rassemble soixantedouze enfants dispersés dans le département par les différents réseaux." (Hazan, 2000, p.116) 
considérant le père de ses enfants inconséquent et trop frivole. Son mari quitte la France, il part vivre aux États-Unis et ne donne plus aucune nouvelle de lui à ses enfants : "Il les a abandonnés".

La relation entre elle et ses enfants est difficile.

Paulette décrit un rapport fusionnel avec sa fille. À l'adolescence, celle-ci va de plus en plus mal, elle présente des "bouffées délirantes" vers l'âge de 18 ans. Puis sa fille met fin à ses jours.

Paulette continue à faire un travail personnel en psychothérapie pendant plusieurs années. Son fils, lui, vit en Israël depuis qu'il a dix-sept ans. Il s'est marié à une Israélienne.

$\mathrm{La}$ relation avec ses petits-enfants est difficile dans la mesure où ils ne parlent pas le français. Leur père ne leur parle qu'en hébreu. C'est sa belle-fille qui a appris le français et qui le parle un peu avec Paulette.

Paulette ne trouve pas sa place de mère auprès de son fils ; elle ne trouve pas non plus sa place de grand-mère auprès de ses petits-enfants.

En 1992, Paulette entend parler de l'association des "Enfants cachés : 1940-1944". Aussitôt, elle y adhère. Paulette dit: "Moi, je suis née à quarante ans, avant je sais même pas comment j'étais". Dès la retraite, Paulette décide de re-parcourir son enfance. Elle se rend dans la Sarthe et part à la recherche des familles qui l'avaient "accueillie".

La relation à ses parents a toujours été conflictuelle: "Ma mère a perdu la tête". Paulette dit qu'elle était dépassée par tout. Son père, qu'elle caractérise de "pervers", interdisait à sa femme de voir sa propre mère. A propos de sa grand-mère, elle dit : "Elle s'est éteinte comme elle a vécu, toute seule". Paulette a appris que sa mère avait subi des expériences médicales dans les camps. Á Bergen-Belsen, elle a été stérilisée. Ses parents sont morts tous les deux en 2001, et à la demande de son fils, ils ont été enterrés à Jérusalem. Paulette dit qu'elle, elle souhaite être enterrée aux côtés de sa fille, dans le cimetière juif de Bagneux. Elle a déjà fait toutes les démarches pour que ce soit possible. 


\section{Analyse}

\section{Un vécu douloureux, une transmission ambivalente du mortifère et des pulsions de vie}

Les débuts de vie de Paulette ont été d'emblée marqués par des séparations, des ruptures, et des abandons. Pour un enfant, la séparation est source de perte et d'angoisse inévitable ici du fait de l'absence de préparation des séparations. En 1942, Paulette avait six ans. Entre six ans et neuf ans, elle a connu l'errance. Elle se décrit comme un enfant qui n'existait pas et que personne ne regardait. Les ruptures brutales sont à l'origine de discontinuité interne avec cette perte de continu d'exister si nécessaire à la construction du Sujet. La problématique du secret est au centre du vécu de Paulette. Le secret imposé à l'enfant et à son environnement constitue un obstacle important à un possible travail d'élaboration. A l'interdit de parler qui lui est imposé, s'ensuit l'interdit de penser.

L'affection que lui a apporté sa grand-mère ainsi que la capacité de Paulette à s'investir au niveau scolaire lui ont permis de se protéger d'une certaine négativité environnante. De par son vécu dans les camps, sa mère était dans une incapacité physique et psychique de prendre soin de sa fille et de la protéger de son père, qui semble-t-il avait eu également un passé douloureux. Le mariage de Paulette a été un échec.

La fille de Paulette a décidé de disparaître réellement au moment de devenir majeure et après avoir vécu, semble-t-il, une relation fusionnelle avec sa mère. La prise d'autonomie fut alors impossible. La singularité de cette relation mèrefille semble illustrer la formule de Carel et Medjahed (2000) à propos de ce type de lien : «Il est incestuel de s'attacher et mortel de se détacher » (p.126).

Le fils de Paulette quitte la France et par conséquent sa mère, un an avant sa propre majorité, comme pour échapper potentiellement à un destin funeste. Il a tenté tout un travail de réaffiliation au groupe juif en choisissant Israël, pays de son père, et en ne parlant que l'hébreu. En outre, il a épousé une $\mathrm{Sabra}^{5}$.

De son côté, Paulette est restée comme suspendue, même si elle aussi, s'est réaffiliée à sa manière, au groupe juif. Elle dit avoir commencé à vivre à l'âge de quarante ans : cet âge

5 Une personne née en Israël. 
6 Le Klezmer est une tradition musicale des Juifs ashkénazes. Elle s'est développée à partir du XVe siècle, ses origines - discutées et dépourvues de sources documentaires d'époque mais vraisemblables seraient les musiques du Moyen-Orient et de Turquie (héritage originel des Ashkénazes descendants des Hébreux en royaume d'Israël), ainsi que les musiques d'Europe centrale et d'Europe de l'Est (Slaves et Tziganes). Le mot klezmer vient de l'association des mots klei et zemer, instrument de chant. À l'origine le mot klezmer (pluriel : klezmorim) désignait donc les instruments. correspond-il à l'âge qu'elle avait au moment du décès de sa fille ? Elle dit qu'à partir de ce moment-là, elle a enclenché la reconstitution de son parcours. Puis au moment de sa retraite, elle est intervenue dans les écoles, pour transmettre son histoire. Elle a participé à une chorale Klezmer $^{6}$, a réappris le yiddish. Mais Paulette n'allait jamais à la synagogue, et ne pratiquait aucune fête. Elle a maintenu un lien avec le monde de ses grands-parents, monde qui a disparu, mais dont les représentants, ses grands-parents, sont les deux seules personnes qui semblent l'avoir maintenue et/ou ramenée à la vie.

\section{Les enfants juifs cachés : une génération au vécu spécifique}

La génération de Paulette est aussi parfois appelée la deuxième génération - par rapport à celle de leurs parents morts dans les camps ou ayant survécu à la Shoah, faisant du génocide un temps premier. Cette deuxième génération est une génération comme sacrifiée dans la mesure où chaque sujet $\mathrm{a}$ été isolé et a tenté, par ses propres moyens, de s'inscrire dans un devenir : fonder une famille et être intégré socialement et professionnellement... souvent dans le silence, jusqu'en 1991, voire 1995 en France.

Dans le même temps, il s'agit d'une génération, qui, par cette inscription a permis l'accès à la vie, car les enfants juifs cachés sont de miraculés, qui, adultes ont donné la vie : ils ont eu des enfants pour la plupart d'entre eux.

On peut donc penser que la génération suivante - dite la troisième : les enfants des enfants juifs cachés - peut également offrir du vivant. C'est le choix qu'a ainsi réalisé le fils de Paulette. En revanche, ce choix a semblé impossible pour sa fille : la mort était la seule issue.

Les miraculés que sont les enfants juifs cachés, car sauvés sans avoir survécu au camp de la mort, représentent la génération qui était désignée pour morte mais qui est restée en vie. Malgré l'intention mortelle, alors internalisée, donner la vie après eux s'est révélé possible. Cette génération est donc celle qui a été témoin de quelque chose qu'il fallait totalement faire disparaître, d'où aussi l'explication que l'on peut donner au silence ou au non-dit pesant qui s'est perpétué. Peut-être est-ce également le sens qui l'on peut attribuer au suicide de 
la fille de Paulette, qui portait en elle, le «fantôme » de la déliaison mortifère.

En outre, de par cette bascule dans la clandestinité en 1942 (année au cours de laquelle est décidée la «solution finale») dans un univers inconnu dans lequel, pour vivre, une nouvelle affiliation s'imposait, ces enfants ont été la cible d'une déculturation qui les a conduits à chercher dans leur devenir adulte, parfois un nouveau groupe ou des groupes. Car dans le processus qui s'est opéré chez ces enfants entre 1942 et 1945, il a été question de désaffilier pour réaffilier, mais cette réaffiliation n'aboutissant pas complètement, a laissé la personne en suspens.

Après la guerre, les procédures de ré-accueil dans le groupe juif initial, n'ont pas pu avoir lieu car le «monde» juif d'avant-guerre avait disparu. Ils ont donc acquis un statut qui les a rendus étrangers à leur propre groupe, à eux-mêmes : ils sont juifs et autre chose. Or le fait même qu'ils soient juifs et qu'ils aient, en eux, des ingrédients d'autres groupes, ne leur a pas permis d'être ou de sentir juifs à part entière. Ils semblent avoir été davantage des vecteurs du judaïsme.

Il est alors possible de penser que cette génération sacrifiée a eu pour fonction de transmettre et de faire perdurer le groupe. Ainsi, intégrer un tel sens dans la construction du récit permet potentiellement à cette deuxième génération de se réaffilier à la chaîne générationnelle : ils sont enfants de leurs parents, et parents de leurs enfants. Le but est de réintégrer leur récit au sein de l'histoire de leur propre lignée. Peut-être serait-ce une piste de soin à penser pour le fils de Paulette qui semble s'être positionné en rupture de sa mère... pour n'être que dans la vie?

\section{Discussion}

“ Un héritage sans testament » (Char, 1967)

Cette situation comme d'autres, montre ainsi l'importance de la filiation et de l'affiliation, qui sont deux dimensions constitutives de l'être humain. La filiation est au cœur de la construction ontologique de tout individu dans la mesure où elle est régie par un principe généalogique "d'appartenance à 
une certaine collectivité constituée par des personnes tenues pour issues d'un ancêtre commun" (Guyotat, 1995, p.8).

Ainsi par la nomination, le nouveau-né est d'emblée inscrit dans la dimension symbolique. Sa naissance se produit nécessairement dans une lignée spécifique qui l'inscrit dans une généalogie, une histoire et une géographie. En tant qu'il est enfant de son père et de $s a$ mère, il est affilié nécessairement à ses filiations : paternelle et maternelle, aux familles qui le portent et au-delà, à ses groupes d'appartenance qui l'introduise dans ses affiliations. D'où l'importance également de l'affiliation, comme socle qui préexiste à tout être humain, avant même sa naissance. Ce terme est emprunté ici à l'anthropologie : il s'agit des appartenances à des groupes (Levi-Strauss, 1973, p.132), qu'ils soient culturels, sociaux, ethniques, religieux... Les affiliations sont plurielles et comme la filiation, ces deux processus ne sont pas des simples processus d'inscription passives, mais des processus interactifs qui supposent une élaboration constante du sujet tout au long de son développement et des différents âges de la vie. Par ailleurs, ils ont tous les deux une dimension consciente, préconsciente et inconsciente.

Par ailleurs, nous identifions deux dimensions inhérentes à la filiation et à l'affiliation : l'individuel et le collectif.

En outre, transmettre l'héritage psychique et culturel des générations qui nous précèdent, à celles qui nous suivent est toujours un processus complexe et délicat. Les bases conceptuelles de la transmission de l'héritage cherchent à repérer les fonctions du groupe familial, qui sont d'une part, de métaboliser les angoisses archaïques du tout-petit, pour amener à la construction d'un monde interne cohérent et rassurant notamment par la transformation des éprouvés sensoriels bruts en vécus psychiques assimilables; d'autre part, d'assurer une fonction de liaison qui donne à l'enfant la possibilité d'utiliser ces vécus psychiques pour s'auto-contenir, organiser sa propre psyché et établir des relations avec les autres. Enfin, avec la fonction de transmission, dans la succession des générations, chaque famille transmet à l'enfant sa façon d'appréhender le monde extérieur et d'organiser son univers interne.

On ne peut pas construire seul son histoire, chacun s'ancre dans une histoire familiale (elle-même ancrée dans sa culture et dans son histoire collective) qui le précède et 
où il puise ses fondations narcissiques et trouve une place de sujet.

Précisons encore ici qu'il existe deux modalités différentes de transmission des héritages (Kaës, 1998). D’une part, l'héritage intergénérationnel qui est constitué de vécus psychiques élaborés mais sans être forcément conscients. Les fantasmes et les identifications organisent une histoire familiale, un récit mythique, où chaque membre de la famille peut puiser les éléments nécessaires à la constitution de son roman familial individuel. C'est ce qui se transmet dans l'ordre du symbolique. D'autre part, l'héritage transgénérationnel qui lui, est constitué d'éléments bruts, non élaborés transmis tels quels. Ce peut être par exemple des secrets, des deuils non faits, des vécus traumatiques: individuels, familiaux, collectifs, etc. Faute d'avoir été élaborés par les générations précédentes, ces éléments bruts font irruption chez les "héritiers" sans qu'ils puissent se les approprier. Ces éléments bruts sont transmis sur un mode projectif, selon une modalité de type suintement émotionnel : l'enfant est le réceptacle des éprouvés des parents, sans en intégrer le sens.

On peut ainsi parfois constater des effets de blocage où la transmission est rejetée car son potentiel mortifère est perçu de manière trop vivace, dangereuse. Un travail thérapeutique sera alors nécessaire pour détoxiquer cette difficile histoire : la proposition consiste à transformer les éléments béta en éléments alpha (Bion, 1962) et peut-être en faire advenir un testament...

\section{Qu'est-ce que cette situation révèle d'un processus actif d'une génération à l'autre?}

Face à la trop forte prégnance des modalités de transmission transgénérationnelle, la transmission traumatique oeuvre en deçà de la représentation et du symbolique. Ces difficultés ont aussi été les conséquences d'un télescopage entre des "événements du présent" et des "événements du passé" mais non mis au passé, avec lequel ils entrent en collusion répétant la rupture des fonctions de protection et empêchant un travail de récit à la fois familial et individuel.

L'effraction liée à ces événements est telle qu'elle met à mal l'enveloppe familiale et culturelle. En outre, lorsque 
la violence de l'histoire collective, comme on l'a vu pour Paulette participe à la dislocation de l'enveloppe culturelle, cela vient alors renforcer le risque de pertes ou de "trous" qui, par les éprouvés bruts déborde les capacités de transformation. Róheim (1943) soulignait toute l'importance pour chacun de la fonction de sécurisation à la culture : "c'est un gigantesque système d'essais plus ou moins heureux pour protéger l'humanité contre le danger de la perte d'objet" (p.63).

Ces vécus bruts et multiples qui n'ont pas été transformés s'accumulent au niveau des strates générationnels, et comportent le risque de se transmettre aux descendants. Ces éléments non élaborés se révèlent par des blancs, des lacunes ou des événements qui paraissent incompréhensibles, voire insensés au regard de l'histoire de l'individu.

Dans cette dynamique de transmission du traumatisme d'une génération à l'autre, Abraham et Torok (1978) évoquent trois processus ayant cours sur trois générations. Le premier concerne "l'indicible » qui conduit à un clivage chez le sujet traumatisé, porteur de la «crypte ». Le second correspond à « l'innommable » à la génération suivante pour laquelle l'événement ne fait l'objet d'aucune représentation verbale, et dont seule l'existence de l'événement est pressentie. Le troisième est « l'impensable » à la génération suivante : le «fantôme » poursuit son travail de déliaison et continue à s'enkyster dans le psychisme familial et individuel. L'existence même du secret est ignorée.

Certains auteurs évoquent la notion de "loyauté invisible", postulant une répétition du même, au gré des générations, qui ne résiste pas à l'épreuve des faits cliniques. "En effet, un enfant qui subit les effets d'un secret parental ou familial, (...), sera un enquêteur actif malgré lui dont le comportement "étrange" constituera une tentative de solution agie à l'expérience insuffisamment symbolisée dont il pressent l'existence chez un parent ou un grand-parent" (Van Heusden et Van Den Eerenbeemt, 1994, p.42). Cette quête peut parfois être épuisante tellement l'investissement psychique est grand et le niveau de transformation est faible, comme ce fut, semble-t-il, le cas pour la fille de Paulette. 


\section{Conclusion}

Les enfants juifs cachés ont dû lutter psychiquement pour survivre dans des conditions aliénantes. Ils se sont trouvés au carrefour d'affiliations en conflit qu'on leur a demandé d'intérioriser. Ils ont dû être hypervigilants pour ne pas trahir, et préserver un secret qu'on ne leur avait pas dit. Ils ont ainsi acquis une hypermaturité pour faire face à l'adversité et continuer de vivre. Mais à quel prix ? Celui de l'angoisse, de la peur, du traumatisme, et de la psychopathologie parfois transmis à leurs descendants. Cependant, ils ont aussi transmis la vie à leurs enfants, devenus l'écho de leur souffrance, révélateurs de leur traumatisme refoulé, mais aussi promesse d'espoir et de renouveau (Feldman, Rottman, 2014).

La transmission de leur histoire d'enfant caché est porteuse d'ambivalence, comme eux, entre perte et sublimation. L'histoire des deux enfants de Paulette en est une parfaite illustration.

La narrativité comme force d'inscription et de liaison permet d'historiciser l'ontogénèse et les interactions du sujet avec son entourage. Il s'agit là de faire en sorte que ces séismes historiques, familiaux et intimes ne se propagent pas aux générations suivantes, que ces lignes de failles puissent être pansées par la métabolisation afin d'empêcher les ruptures transgénérationnelles. On voit bien comment ces traumatismes s'apparentent à des secousses sismiques psychiques dans la subjectivité des Sujets et de leurs descendants.

Paul Ricoeur (1994) a bien montré comment la privation narrative constituait une souffrance de dire.

Abraham N., Torok M. (1978). L'écorce et le noyau, Paris, Flammarion, 2001.

Bion W. (1962). Aux sources de l'expérience, Paris, Puf, 1979.

Références bibliographiques

Carel A., Medjahed M. (2000). Le traitement à domicile. Une forme de thérapie familiale, Groupal, Vol. 6, pp.123-142.

CHAR R. (1967). Fureur et mystère, Paris, Flammarion.

Conan E., Rousseau H. (1994). Un passé qui ne passe pas, Paris, Fayard.

Dwork D. (1991). Children with a star: Jewish Youth in Nazi Europe, New York, Vail-Ballou press.

Feldman M. (2019). Des traces indélébiles de traumas cachés, Psychothérapies, 39, Vol. 2, pp.71-77. 
Feldman M. (2016). Sauvés mais «exposés ». Impacts de traumas d'enfance tardivement reconnus. A propos des enfants juifs cachés en France pendant la Seconde Guerre mondiale, in Les enfants exposés aux violences collectives, pp.59-71. Toulouse, Erès.

Feldman M. (2009), Entre trauma et protection : quel devenir pour les enfants juifs cachés en France (1940-1944) ? Toulouse, Erès.

Feldman M., Rottman H. (2014). Du vécu au perçu : à propos des enfants juifs cachés en France pendant l'Occupation et de la descendance, in Bantman P., Une génération, l'autre, pp.119-134. Paris, In Press.

Guyotat J. (1995). Filiation et puerpéralité. Logiques du lien, Paris, Puf.

Hazan K. (2000). Les orphelins de la Shoah, les maisons de l'espoir, Paris, Belles Lettres.

Huston N. (2006). Lignes de failles, Arles, Actes Sud.

KAËS R. (1998). Différences culturelles et souffrances de l'identité, Paris, Dunod.

Khan M.R. (1976). Le soi caché, Paris, Gallimard.

Levi-Strauss C. (1973). Organisation sociale. In: Anthropologie structurale deux, pp.89-135. Paris, Plon, 2010.

Poujol C. (2006). L'Affaire Finaly, les enfants cachés, Paris, Berg international.

Ricoeur P. (1994). "La souffrance n'est pas la douleur", in Von Kaenel J.-M., Ajchenbaum-Boffety B., Souffrances, corps et âme, épreuves partagées, Autrement, $\mathrm{n}^{\circ} 142$, pp.58-70.

RoheIm G. (1943). Origine et fonction de la culture, Paris, Gallimard, 1972.

Tisseron S. (1995). Les images psychiques entre les générations, in Le psychisme à l'épreuve des générations : clinique du fantôme, pp.123-144. Paris, Dunod.

Van Heusden A., Van Den Eerenbeemt E. (1994). Thérapie familiale et générations, Paris, Nodules Puf, pp.29-119. 